\title{
Design a model of Image Restoration using AI in Digital Image Processing
}

\author{
Boosi Shyamala ${ }^{1}$, Dr. Chetana Tukkoji ${ }^{2}$, Archana S Nadhan ${ }^{3}$, Dioline Sara $^{4}$ \\ 1,2,3 Assistant Professor, Department of CSE, GITAM School of Technology, GITAM Deemed to be University, \\ Bangalore \\ ${ }^{4}$ Assistant Professor, Department of EECE, GITAM School of Technology, GITAM Deemed to be University, \\ Bangalore
}

Article History: Received: 11 January 2021; Accepted: 27 February 2021; Published online: 5 April 2021

\begin{abstract}
Image restoration is the process of obtaining a distorted/noise image and giving an approximate clear image of the original image. False focus, motion blur and noise are forms of distortion. Image restoration can be done by reversing the process called Point Extension Function (PSF). In this process, the blurred image is generated by point source imaging and can be used to restore the image lost due to the blur process. Like to form. Modern artificial intelligence (AI) applied to image processing includes facial recognition, object recognition and detection, video, image action, and visual search. It helps to develop smart applications in digital image processing.
\end{abstract}

Keywords: PSF, AI, Image Restoration.

\section{Introduction}

Artificial Intelligence has grown to a wide range in these years. Major branch of it is image processing. The main field in which the AI is implemented is image restoration and is mainly used in the field of security surveillance, systems in healthy department, medical diagnosis, military etc.

Reconstruction of 3D objects and more and more researches on their applications. The input data is obtained through different technologies and equipment, and is calculated based on image processing technology, geometric modeling and computer graphics. The 3D model is the output data, showing the initial shape of the real object. In this study, 3D objects were reconstructed using RGB-D cameras. Check and analyze different methods of recreating 3D objects from a set of 3D point clouds of different devices. Based on a combination of image processing and geometric modeling, create suggested 3D model objects from a set of 3D point clouds. Compare the results obtained and apply them to various methods to find the most suitable method to obtain noisy real data from Microsoft kinect in the most accurate and dynamic way.

Handwritten character recognition is one of the most important means to automatically translate important historical documents in digital libraries. This is a difficult task because it requires a set of labor-intensive manual functions for character classes. The intra-class variability of handwritten characters is the basic bottleneck of accuracy recognition. You can see Convolutional Neural Networks (DCNN) in this article, which provides an indepth introduction to handwritten character recognition in Kannada manuscripts. The DCNN model combines feature extraction and classification. Learn the most unique features from the given data. Therefore, the use of manual functions is reversed. This is the unified feature classification and derivation of the DCNN model. In this case, due to its most unique quality, the provided data can be learned and manual functions can be reversed. Use DCNN characters to input the extracted features into SVM and SGDM classification algorithms for recognition. This has been tested and belongs to the Kannada stone sculpture in the 11th century, and promising results have been observed in numerical estimation [1].

\section{Literature}

There are a lot of resemblances in different brands to accomplish the difficulty to fix the brand of Automatic identification. Identification of Volkswagen vehicle new system is developed in this work. Firstly, by the manual revision and watershed algorithm the original image of the car were pre-processed. Secondly the wavelet entropy (WE) is employed in the car images to extract efficient characteristics. Thirdly, we used a classifier called multilayer perceptron (MLP). At last, algorithm used is artificial bee colony (ABC) for training the MLP. The original $\mathrm{ABC}$ is poor at exploitation due to its equation system where as good at exploration. So, improved artificial bee colony (IABC) is proposed as a new model to balance the exploitation and exploration of ABC. For fair comparison we used the validation 5 X 5 fold cross. The result of the experiment showed that $88.62 \%$ is the overall specificity, $89.17 \%$ is the overall sensitivity and $89.17 \%$ is the overall accuracy. Therefore for Volkswagen vehicle identification the proposed method is effective. The ordinary ABC performance is not better as IABC [2].

IoT-based systems and computer vision plays a important role in home security field. In this paper, to minimize the threat made due to burglary IoT based home security is designed and implemented with integrated intrusion detection. Combination of web application and web server proposed system to control their status to remotely 
access. This system efficiently and effectively monitors the home automatically which includes images that process faster using an real time identification system.

The main purpose of this article is to define faces to ensure family safety. Raspberry Pi is a single-board computer in which images can be captured to identify and detect images in the system. The Haar cascade algorithm is used in this project to use the LBPH algorithm for face detection and facial recognition, and a simplified version of Raspberry Pi's SQL (called SQLite) is used with MYSQL for the web server to update MYSQL. Finally, use Twillo (Internet of Things application) to send notifications and visual effects to users via SMS. The results obtained according to the research will be used as a real-time system and can be used without any manual intervention. The system includes efficient use, convenient user service and instant access [4].

In image processing image segmentation is a very important problem which has a vast range of applicability in biomedicine, medicine and more others in the field of engineering and science. Based on techniques of calculus of variation and partial differential equations the non-learning-based approaches have acquires many achievements and lot of attentions have been attracted. The well known model which is used to solve image segmentation problem is Chan-Vese variational segmentation. The highly accurate method is the level set method used to solve this model and doesn't depend on the edges. However, these methods performance depends on level set function and the initial boundary also. In this paper work, depends on edge detector automatic initial boundary generation were proposed by Canny, Roberts, Sobel, and Prewitt. Four proposed methods of initial boundary generation is proved based on method of Canny's edge detector to segment biomedical images by implementing Chan-Vese model. Compare different edge and improved segmentation results were obtained segmentation results by experiments [5].

To monitor the events nowadays we widely use visual surveillance system effectively analysing the behaviour and the crowd in the challenging event. Analysing and better understanding the crowd behaviour is considered as the basic element of the group. To distinguish the individuals and groups the crowded scene is the exigent task. In this paper a video based framework is proposed that the group of people efficiently identified from the crowd. The contour in literature is a group of extraction boundary composed the framework. ViBe is a background subtraction algorithm is a proposed to make care of where the relevant features are extracted and contours incurred by video framing. By calculate the distance and area by further detecting the crowd in a group by threshold frames. The university campus has analysed a self gathered dataset. An average 86.065accuracy is not distinguished by the proposed frame work group [6].

An image retrieval method for textile industry database based on multiple image features of CBIR system is proposed. The combination of various well-structured shapes, colors and textures are the characteristics of the various functional technologies included. Nowadays, CBIR systems mostly use queries in an exemplary manner, in which suggested images are extracted from the database for similar feature queries.

In image retrieval a particular image is classified in the category belonging to the traditional, a large image datasets are classified by a lot of approaches. For getting the desired results in the first go users wanted the real world applications as traditional CBIR techniques fail. Learning model is Support vector machine with retrieval process which when used to find images more often appropriately. Also, the size is not so large for the dataset even when it is useful. The CBIR system is retrieved using SVM classifier, from the database of the more relevant images. In this paper, the classification of the images and the capability of SVM is evaluated [7].

\section{Comparison}

A method in Image Processing is to perform operators on images to extract some important information to relieve an enhanced image. A method of signal processing by which the input as well as output features/characteristics of the images which is associated. In this paper point processing technique is used by us because without the knowledge of the surrounding, change of each pixels grey value taken place. The arithmetic operation is performed in order to process each points by applying simple function $\mathrm{u}=\mathrm{f}(\mathrm{x})$ to the pixel values. The given images original points are reconstructed along with arithmetic operations to complement and can also be applied.

In this paper threshold and histogram methods are used in the segmentation operations which separate the background noise from the image and to increase the thickness of image. Some methods of filtering methods used are used like Frequency High and Low pass filter, Spatial and Gaussian Filter, from reconstructed the image to remove [8].

This paper uses a multi-mode medical image segmentation method to extract different indicators of the image fusion network strategy, merges the multi-channel images channel by channel into a multi-channel input, learns the fusion feature representation, and then trains the segmentation network to obtain better accuracy. For multimode medical image segmentation, the fusion strategy plays an important role in achieving accurate segmentation results.

Choosing an effective deep learning fusion strategy is still an important issue. In the 2013-2018 BraTS Challenge, all methods applied input-level fusion to directly integrate different MR images in the input space, which is simple and retains the inherent image characteristics, and allows the method to focus on the follow-up The segmentation design network architecture, such as multi-task, multi-view, multi-scale and GAN-based 
strategies. The pre-processing and post-processing methods we use can be applied to the segmentation part of the image [9].

The properties of nanoparticles are related to their size, size distribution and shape. In the field of nanoscience, accurate measurement of these properties is essential. In this paper, ultrasonic spray pyrolysis (USP) was used to synthesize silver nanoparticles (AgNPs) from silver nitrate aqueous solution. The synthesized AgNP was characterized by dynamic light scattering (DLS) analysis and scanning electron microscope (SEM) to reveal its size and size distribution. Sub-images are created based on user-defined maximum and minimum search granularity criteria. The search particle algorithm aims to determine whether there are nanoparticles in the subimage, and if the algorithm finds one or more particles, it will mark its diameter and location.

The Gaussian noise algorithm is used to remove the noise in the sub-image. We have used histogram and threshold methods to find the dark and light values of each pixel in the sub-image to reconstruct the original image [10].

In this paper bounded variation function is used to reconstruct the original image BV of functions of bounded variation is used for double phase functional in the space. The classical ROF-model for image restoration calls for minimizing the energy. Mathematical theorems are applied along with BV function with some assumption [11].

In recent years, in medical image segmentation, convolutional neural networks have begun to surpass classic statistical data and machine learning techniques based on atlases, and have been proven to be excellent in performance and speed. CNN's evaluation of data collected under different acquisition conditions (for example, scanner type, sequence type, sequence parameters) as training data shows that there is a dependency between the intensity normalization method and CNN segmentation performance [12].

\section{Architecture:}

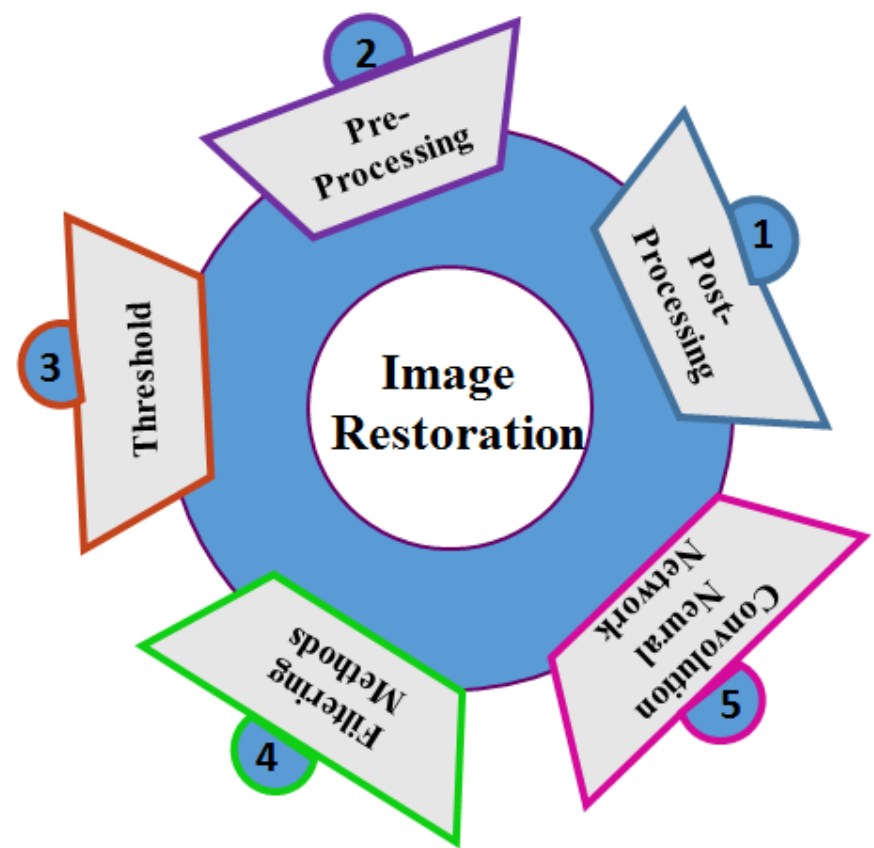

Figure 1. Architecture

The figure 1 show the architecture of the Image Restoration.Post processing is the method of editing the data collected by the camera when taking the picture to improve the object. Better data captured during the click of a photo is the likelihood of change. There are more and more cameras on the market that can record RAW images.Image pre-processing is the term for the lower abstraction image operations that are intended to enhance the image data to eliminate unnecessary distortions or increase those image characteristics essential to further processing. Pre-processing image usage redundancy in pictures.Contrast adjustment, intensity adjustment, histogram equalization, binarization and morphological operation are compared among pre-processing methods. The threshold is a kind of image segmentation where the pixels of an image are changed in order to allow analysis of the image. At thresholding, the picture is transformed from color or grey into a binary picture, that is, a black and white picture.Filtering is an image manipulation or enhancement process. For example, to highlight those features, you can select an image or delete other features. Filtering image processing operations include smoothing, sharpening and edge improvement. In supervised learning techniques one of the best methods is Convolution Neural Networks has played vital role for the speech recognition and image processing. 


\section{Conclusion}

In high-level vision issues, such as object recognition, convoluted networks have experienced tremendous success. The effect may be used to assist human perception or as an input to other tasks such as identification and segmentation of a machine vision.

\section{References:}

1. "A Research for Reconstructing 3D an Object by Using RGB-D Camera", Van Sinh Nguyen, Manh Ha Tran, and Quang Minh Anh Le.

2. "Deep Convolutional Neural Networks for Recognition of Historical Hand written Kannada Characters", H. T. Chandrakala and G. Thippeswamy.

3. "Volkswagen Vehicle Identification via Multilayer Perceptron Trained by Improved Artificial Bee Colony Algorithm", Jingyuan Yang, Lei Wang, and Qiaoyong Jiang.

4. “A Low-Cost Internet of Things-Based HomeSecurity System Using Computer Vision”,Hasan Salman, Md. Arifur Rahman Nayeem, Asif Mohammad,Xiaodong Bai, Md. Rezwan Mamun, Md. Mamun Ali, and AsaduzzamanPeol.

5. "Automatic Initial Boundary GenerationMethods Based on Edge Detectors for the LevelSet Function of the Chan-VeseSegmentationModel and Applications in Biomedical ImageProcessing”, Dang N. H. Thanh, Nguyen Ngoc Hien, V. B. Surya Prasath, Le Thi Thanh, and Nguyen Hoang Hai.

6. "An Efficient Vision-Based Group DetectionFramework in Crowded Scene", Monika Pandey(\&), Shivam Singhal, and Vikas Tripathi.

7. "Content-Based Image Retrieval for TextileDataset and Classification of Fabric TypeUsing SVM", Chirag Arora, V. Vijayarajan, and R. Padmapriya.

8. Multimedia Image Processing Lab Experiment/Simulation, Md Mamunur Rashid, American International Journal of Sciences and Engineering Research; Vol. 3, No. 1; 2020 ISSN 2641-0303 E- ISSN 2641-0311 Published by American Center of Science and Education, USA.

9. “A review: Deep learning for medical image segmentation using multi-modality fusion", Tongxue Zhou, SuRuan, St_ephaneCanu, www.elsevier.com/journals/array/2590-0056/open-access-journal, 2019.

10. "New Proposal for Size and Size-DistributionEvaluation of Nanoparticles Synthesized via Ultrasonic Spray Pyrolysis Using Search Algorithm Based on Image-Processing Technique”, Elif Emil Kaya, Ozan Kaya, GözdeAlkan, SebahattinGürmen, SreckoStopic and Bernd Friedrich, www.mdpi.com/journal/materials,doi:10.3390/ma13010038, 2020.

11. "Double phase image restoration", PetteriHarjulehto, Peter Hästo,, Journal of Mathematical Analysis and Applications, Sep 2019.

12. "Analysis of intensity normalization for optimal segmentationperformance of a fully convolutional neural network”, Nina Jacobsen, Andreas Deistung, Dagmar Timmann, Sophia L. Goericke, Jürgen R. Reichenbach, Daniel Güllmar, November 2018. 